\title{
Multi-way analysis of diversity and redundancy factors in large MOX gas sensor data
}

\author{
L. Fernandez ${ }^{1,2}$, A. Gutierrez-Galvez ${ }^{1,2}$, S. Marco ${ }^{1,2}$, \\ ${ }^{1}$ Institute for Bioengineering of Catalonia (IBEC). Baldiri Reixac 4-8, 08028 Barcelona, Spain \\ ${ }^{2}$ Department of Electronics, University of Barcelona, Martí i Franqués 1, 08028-Barcelona, Spain \\ Ifernandez@el.ub.es
}

\begin{abstract}
We propose the use of multi-way methods to analyze the contribution of diversity and redundancy to odor identification and concentration estimation in a large chemical sensor array. We use a chemical sensing system based on a large array of metal oxide sensors (MOX) and inspired on the diversity and redundancy of the olfactory epithelium. In order to analyze the role of diversity (different sensor type and temperature modulation) and redundancy (replicates of sensors and different load resistors) in odor quantification and discrimination tasks, we have acquired two datasets and modeled the data using multi-way techniques.
\end{abstract}

Key words: Artificial Olfaction, large array ,MOX gas sensor, multi-way methods.

\section{Introduction}

The olfactory epithelium is populated by millions of olfactory receptors neurons (ORNs) that bind to a number of odor molecules. When exposed to a variety of odorants, ORNs of different type show differences in their activation patterns [1], so that sensory diversity encodes the odor quality. When exposed to a single odorant, ORNs of the same family present an almost continuous variation of their dose-response curves [2], thus sensory redundancy allows the encoding of odor quantity.

\section{Methods}

Taking inspiration from the ORN population in the olfactory epithelium, we have built a large MOX sensor array that combines sensor diversity by means of 10 different sensor families modulated in temperature [3], and sensor redundancy with sensor replicates and different load resistors. A total of 96 MOX Figaro and FIS commercial sensors placed in 8 identical and independent compartments plus one temperature and one humidity sensor per compartment. A module with two high-speed multiplexors (NI PXI 2530) allows switching the connection of the sensors to 16 load resistors $(100 \Omega-100 \mathrm{~K} \Omega)$ to measure the sensor output.

Using this system, we have collected two datasets. Dataset I was designed to test the system in odor discrimination tasks, and consisted in the binary mixtures of 3 odorants ethanol, acetone, butanone). The experiments were designed to have a transition from a first analyte to a second analyte by decreasing the concentration of the first analyte and increasing the concentration of the second in six steps. This procedure is repeated for the 3 binary combinations of the 3 analytes for a total of 18 experiments (Table I). Dataset II was devised for odor quantification purposes, and comprised 7 different concentrations of ethanol $(0,20$ $40,60,80,100$ and $120 \mathrm{ppm}$ ). The experiments were randomized for the collection. The complete set of experiments was repeated 7 times for dataset I and 9 for dataset II.

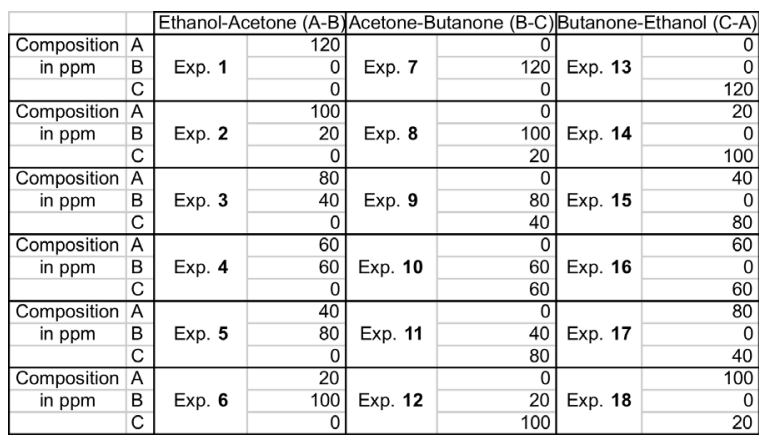

Table. 1: Table of experiments of dataset I. Three binary mixtures from ethanol, acetone and butanone with 6 different concentration ratios each. 


\section{Results}

The datasets are analyzed using parafac, a multiway method that allows separating the contributions in odor identification and concentration estimations of each of the modes used. In our study the modes used are sensor type, temperature, load resistor, and samples. Figure 1 shows the sample scores and the corresponding loadings for temperature, resistor and sensor variables of the parafac model built with a single repetition of the 6 mixtures of ethanol and acetone in dataset $I$, for 8 temperature modulated sensors with all 16 load resistors.
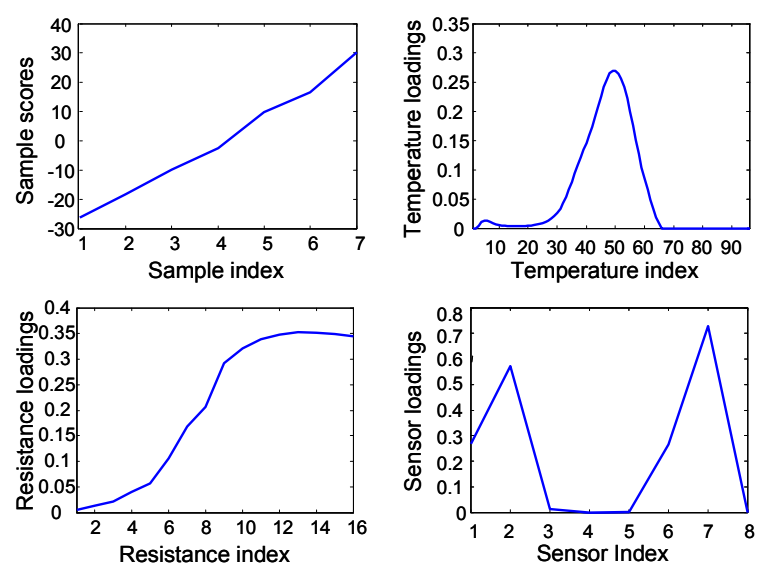

Fig. 1: Plots of sample scores and loadings for the 1 component Parafac model corresponding to 6 binary mixtures of ethanol and acetone (dataset I). Sample scores show a clear linear tendency. Important loading contributions can be found at medium temperatures, high resistances and sensor families 2 a 7 (TGS-2600, TGS 2630).

Figure 2 shows the predicted concentration of ethanol and the mean square error in prediction for a NPLS built using the 8th first repetitions of dataset II for calibration and the last one as validation subset, using the complete set of variables.

Pred vs. fitted by NPLS 4 LV - RMSEP $=4.77$

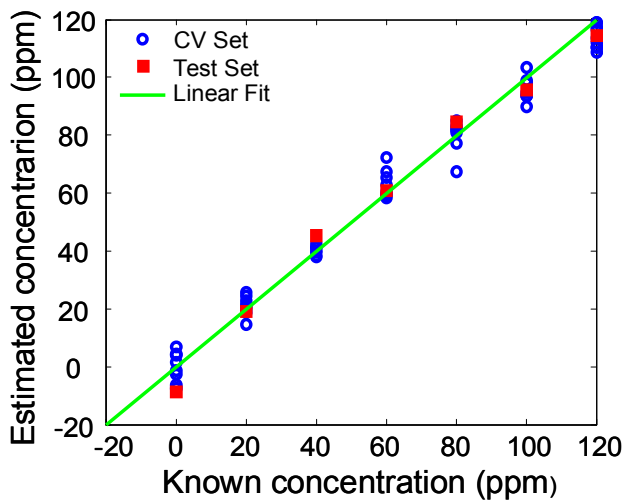

Fig. 2: Plot of predicted vs fitted scores of the 4 latent latent variable $\mathrm{npls}$ model corresponding to 7 ethanol concetrations (dataset II).

\section{Conclusions}

We presented an analysis of sensory diversity and redundancy for a large MOX sensor array. We showed that multi-way techniques simplify data interpretation since they separate the loadings for each mode, also reducing significantly their number.

\section{Acknowledgements}

The research leading to these results has received funding from the European Community's Seventh Framework Programme (FP7/2007-2013) under grant agreement no.216916.

The authors are from a consolidated Grup de Recerca de la Generalitat de Catalunya and has support from the Departament d'Universitats, Recerca I Societat de la Informació la Generalitat de Catalunya (expedient 2009 SGR 0753).

\section{References}

[1] Malnic B, Hirono J, Sato T, Buck L, Combinatorial receptor codes for odors. Cell (1999) vol. 96 (5) pp. 713-723

[2] Grosmaitre X, Vassalli A, Mombaerts P,Shepherd G, Ma, M, Odorant responses of olfactory sensory neurons expressing the odorant receptor MOR23: a patch clamp analysis in gene-targeted mice. Proceedings of the National Academy of Sciences (2006) vol. 103 (6) pp. 1970-1975

[3] Gutierrez-Osuna R, Gutierrez-Galvez A, Powar $\mathrm{N}$, Transient response analysis for temperaturemodulated chemoresistors. Sensors \& Actuators: B. Chemical (2003) vol. 93 (1-3) pp. 57-66 